\title{
OFF-DIAGONAL ESTIMATES OF SOME BERGMAN-TYPE OPERATORS ON TUBE DOMAINS OVER SYMMETRIC CONES.
}

\author{
CYRILLE NANA AND BENOÎT F. SEHBA
}

\begin{abstract}
We obtain some necessary and sufficient conditions for the boundedness of a family of positive operators defined on symmetric cones, we then deduce off-diagonal boundedness of associated Bergman-type operators in tube domains over symmetric cones.
\end{abstract}

\section{INTRODUCTION}

The Bergman projection is the orthogonal projection from the Lebesgue space $L^{2}$ onto its closed subspace consisting of holomorphic functions. In this note, we obtain some answers on the question of the boundedness of the Bergman projection from the Lebesgue space $L^{p}$ to $L^{q}$ when $1 \leq p \leq q \leq \infty$ in the setting of tube domains over symmetric cones. For this, we first study a family of positive operators defined on symmetric cones. For our purpose, we introduce some definitions and notations.

Let $\Omega$ be an irreducible symmetric cone in $\mathbb{R}^{n}$. There are a notion of rank and a notion of determinant function associated to each symmetric cone (see [14]). We write $r$ and $\Delta$ for the rank and the determinant function associated to $\Omega$ respectively. We recall that for $n \geq 3$, the Lorentz cone $\Lambda_{n}$ of $\mathbb{R}^{n}$ is the set defined by

$$
\Lambda_{n}=\left\{\left(y_{1}, \cdots, y_{n}\right) \in \mathbb{R}^{n}: y_{1}^{2}-\cdots-y_{n}^{2}>0, y_{1}>0\right\}
$$

it is an example of symmetric cone of rank $r=2$ and its determinant function is given by the Lorentz form

$$
\Delta(y)=y_{1}^{2}-\cdots-y_{n}^{2} .
$$

For $1 \leq p<\infty$ and $\nu \in \mathbb{R}$, we write $L_{\nu}^{p}(\Omega)=L^{p}\left(\Omega, \Delta^{\nu-\frac{n}{r}}(y) d y\right)$ for the space of functions $f$ defined on $\Omega$ that satisfy

$$
\|f\|_{p, \nu}=\|f\|_{L_{\nu}^{p}(\Omega)}:=\left(\int_{\Omega}|f(y)|^{p} \Delta^{\nu-\frac{n}{r}}(y) d y\right)^{1 / p}<\infty .
$$

Let $\alpha, \beta, \gamma$ be real parameters. We consider the integral operators $S=S_{\alpha, \beta, \gamma}$ which are defined on $\Omega$ by

$$
S g(y)=\Delta^{\alpha}(y) \int_{\Omega} \Delta^{-\gamma}(y+x) g(x) \Delta^{\beta}(x) d x
$$

The above family can be seen as a generalization of the Hilbert-type operators considered in [1]. It has been considered in several works related to the question of boundedness of Bergman operators (see for example $[3,4,19]$ and the references therein).

2000 MATH SUBJECT CLASSIFICATION: 42B35, 32M15.

KEYWORDS: BERGMAN PROJECTION, SYMMETRIC CONE. 
In the first part of this paper, we consider the question of off-diagonal boundedness of the family $S_{\alpha, \beta, \gamma}$, that is its boundedness from $L_{\nu}^{p}(\Omega)$ to $L_{\mu}^{q}(\Omega)$ for $1 \leq p \leq q \leq \infty, \nu, \mu \in \mathbb{R}$.

One application of the study of the family $S_{\alpha, \beta, \gamma}$ (see for example [4, 19]) is that its boundedness implies the boundedness of a related family of Bergman-type operators defined below.

For $\Omega$ an irreducible symmetric cone in $\mathbb{R}^{n}$, we write $\mathcal{D}=\mathbb{R}^{n}+i \Omega$ for the tube domain over the cone $\Omega$. Let us denote by $\mathcal{H}(\mathcal{D})$ the space of holomorphic functions on $\mathcal{D}$. For $1 \leq p<\infty$ and $\nu \in \mathbb{R}$, we write $L_{\nu}^{p}(\mathcal{D})=L^{p}\left(\mathcal{D}, \Delta^{\nu-\frac{n}{r}}(y) d x d y\right)$ for the space of functions $f$ in $\mathcal{D}$ satisfying

$$
\|f\|_{p, \nu}=\|f\|_{L_{\nu}^{p}(\mathcal{D})}:=\left(\int_{\mathcal{D}}|f(x+i y)|^{p} \Delta^{\nu-\frac{n}{r}}(y) d x d y\right)^{1 / p}<\infty .
$$

The weighted Bergman space is the set $A_{\nu}^{p}(\mathcal{D})=L_{\nu}^{p}(\mathcal{D}) \cap \mathcal{H}(\mathcal{D})$. It is a closed subspace of $L_{\nu}^{p}(\mathcal{D})$ and it is well known that it is nontrivial only for $\nu>\frac{n}{r}-1$ (see [13]). The usual Bergman space $A^{p}(\mathcal{D})$ corresponds to the case $\nu=\frac{n}{r}$.

We also consider the mixed norm version of the above spaces. For $1 \leq p, q \leq \infty$, we write $L_{\nu}^{p, q}(\mathcal{D})=L^{p, q}\left(\mathcal{D}, \Delta^{\nu-\frac{n}{r}}(y) d x d y\right)$ for the space of functions $f$ in $\mathcal{D}$ satisfying

$$
\|f\|_{p, q, \nu}=\|f\|_{L_{\nu}^{p, q}(\mathcal{D})}:=\left(\int_{\Omega}\left(\int_{\mathbb{R}^{n}}|f(x+i y)|^{p} d x\right)^{q / p} \Delta^{\nu-\frac{n}{r}}(y) d y\right)^{1 / q}<\infty
$$

for $1 \leq q<\infty$, and

$$
\|f\|_{p, \infty}=\|f\|_{L^{p, \infty}(\mathcal{D})}:=\sup _{y \in \Omega}\left(\int_{\mathbb{R}^{n}}|f(x+i y)|^{p} d x\right)^{1 / p}<\infty .
$$

The mixed norm Bergman space $A_{\nu}^{p, q}(\mathcal{D})$ is defined as above. Note that the space $A^{p, \infty}(\mathcal{D})$ is the Hardy space $\mathcal{H}^{p}(\mathcal{D})$.

The Bergman projection $P_{\nu}$ is the orthogonal projection mapping $L_{\nu}^{2}(\mathcal{D})$ onto $A_{\nu}^{2}(\mathcal{D})$. It is defined by

$$
P_{\nu} f(z)=\int_{\mathcal{D}} B_{\nu}(z, w) f(w) d V_{\nu}(w)
$$

where

$$
B_{\nu}(z, w)=d_{\nu} \Delta^{-\nu-\frac{n}{r}}\left(\frac{z-\bar{w}}{i}\right)
$$

is the weighted Bergman kernel and $d V_{\nu}(w)=\Delta^{\nu-\frac{n}{r}}(\Im w) d V(w), d V$ being the Lebesgue measure on $\mathbb{C}^{n}$ (see [4]). The question of whether $P_{\nu}$ extends or not as a bounded operator on $L_{\nu}^{p}(\mathcal{D})$ for $p \neq 2$ has attracted a lot of attention in recent years (see $[2,3,4,5,6,10,11,13]$ and the references therein). Only the case of the tube domains over the Lorentz cone has been completely settled after the works $[5,6,7,15]$ (cf. also [4] and [10]), and the recent proof of the $l^{2}$-decoupling conjecture by Bourgain and Demeter [12] used as a key tool in [11] and [8]; note that authors of [8] present the complete answer to this question for the case of the Pyateckii-Shapiro domain, which is a Siegel domain of type II associated to the spherical cone of $\mathbb{R}^{3}$. 
In the second part of this work, we consider the same question but on the off-diagonal side for a family of operators generalizing the Bergman projection. This family is given by the integral operators $T=T_{\alpha, \beta, \gamma}$ and $T^{+}=T_{\alpha, \beta, \gamma}^{+}$defined for functions in $C_{c}^{\infty}(\mathcal{D})$ by the formula

$$
T f(z)=\Delta^{\alpha}(\Im z) \int_{\mathcal{D}} B_{\gamma}(z, w) f(w) \Delta^{\beta}(\Im w) d V(w)
$$

and

$$
T^{+} f(z)=\Delta^{\alpha}(\Im z) \int_{\mathcal{D}}\left|B_{\gamma}(z, w)\right| f(w) \Delta^{\beta}(\Im w) d V(w) .
$$

We note that the boundedness of $T^{+}$implies the boundedness of $T$ and the reverse is not necessarily true. It is clear that estimates obtained for this family of operators imply corresponding estimates for the weighted Bergman projection since $P_{\nu}=T_{0, \nu-\frac{n}{r}, \nu}$ for all $\nu>\frac{n}{r}-1$. The $L_{\nu}^{p, q}(\mathcal{D})$-boundedness of this family of operators has been considered in [5] for the case $T_{0, \mu-\frac{n}{r}, \mu}$ and in [3] for $T_{0, \mu-\frac{n}{r}, \mu+m}$ when $\Omega$ is the light cone. Also, the second author in [19, Theorem 1.1] considered this family and obtained $L_{\nu}^{p, q}(\mathcal{D})$ optimal results for the operator $T^{+}$. In these works, was used the Schur Lemma with test functions which are generalized powers of the determinant function. Moreover, authors of [8] extended some results of [7] to the case of homogeneous Siegel domains of $\mathbb{C}^{n}$ of type II. They obtained sufficient conditions for the operator $T_{\alpha, \mu-\tau, \mu+\alpha}^{+}$in the vectorial weighted case, using the Schur Lemma. As was indicated in the introduction of [16] and explicitly presented in [17], it is not yet known in general, with the techniques developed so far whether these sufficient conditions are necessary. However, with some restrictions on the weight or the choice of the cone (the Vinberg cone for example [9]), these sufficient conditions are necessary.

We note that in [19], the estimates for the positive operators $T_{\alpha, \beta, \gamma}$ were applied in the characterization of the dual space of a Bergman space in the case where the associated Bergman projection is not necessarily bounded. Also, authors of [6] made a heavy use of the family $T_{\alpha, \nu-\frac{n}{r}, \nu+\alpha}$ in their characterization of Besov spaces of tube domains over symmetric cones.

The aim of this paper is to extend to the setting of the tube domains over symmetric cones, the $L^{p}-L^{q}$ estimates of the Bergman projector, when $1 \leq p \leq q \leq \infty$, considered by authors of [1], who studied the case of upper half plane. This leads us to more general result than those of [19]. Our motivation is actually to know whether the Okikiolu test [18], which is a generalization of Schur test, used so far in various papers, is extendable in higher ranks. As we said earlier, it has been noticed recently that in vectorial weighted cases, it is not clear that the Schur Lemma gives sharp results (see [13],[17] for example). The techniques that we develop here, do not impose the use of the generalized powers of the determinant function as test functions. Actually, our results lead to necessary and sufficient conditions for the $L^{p, q}(\mathcal{D})$-boundedness of the Bergman operator with positive Bergman kernel $P_{\nu}^{+}$(see Corollary 5.1 in the text), that coincide with classical ones obtained by the Schur Lemma via generalized powers of the determinant function as test functions.

This work is divided in 5 sections. In Section 2, we state our results; in Section 3, we recall some of the key tools needed to establish our results; in Section 4 , we prove off-diagonal estimates for the operator $S_{\alpha, \beta, \gamma}$ and the proofs of the off-diagonal estimates for the family of Bergman-type operators $T_{\alpha, \beta, \gamma}$ are given in Section 5 . 


\section{Statement of the Results}

2.1. Positive operators on the cone. The following result provides the right relations between the parameters under which the operators $S_{\alpha, \beta, \gamma}$ are bounded.

Theorem 2.1. Let $\nu, \mu \in \mathbb{R}$ and $1<p \leq q<\infty$. Assume that $\frac{\nu}{p^{\prime}}+\frac{\mu}{q}>0$. Then the following conditions are equivalent:

(a) The operator $S$ is bounded from $L_{\nu}^{p}(\Omega)$ into $L_{\mu}^{q}(\Omega)$.

(b) The parameters satisfy

$$
\gamma=\alpha+\beta+\frac{n}{r}-\frac{\nu}{p}+\frac{\mu}{q}
$$

and

$$
p\left(\beta-\gamma+2 \frac{n}{r}-1\right)-\frac{n}{r}+1<\nu<p(\beta+1)+\frac{n}{r}-1,
$$

and

$$
\frac{n}{r}-1-q \alpha<\mu<q(\gamma-\alpha)-\frac{n}{r}+1
$$

We also obtain the following first limit case.

Theorem 2.2. Let $\nu, \mu \in \mathbb{R}$ and $1<q<\infty$. Assume that $\mu>0$. Then the following conditions are equivalent:

(a) The operator $S$ is bounded from bounded from $L_{\nu}^{1}(\Omega)$ into $L_{\mu}^{q}(\Omega)$.

(b) The parameters satisfy

$$
\gamma=\alpha+\beta+\frac{n}{r}-\nu+\frac{\mu}{q}
$$

and

$$
\gamma>0
$$

and

$$
\frac{n}{r}-1-q \alpha<\mu<q(\gamma-\alpha)-\frac{n}{r}+1
$$

The following second limit case is also proved.

Theorem 2.3. Suppose $\nu \in \mathbb{R}$, and $1<p<\infty$. Then the following conditions are equivalent:

(a) The operator $S$ is bounded from $L_{\nu}^{p}(\Omega)$ to $L^{\infty}(\Omega)$.

(b) The parameters satisfy

$$
\gamma=\alpha+\beta+\frac{n}{r}-\frac{\nu}{p}
$$

and

$$
\nu<p(\beta+1)+\frac{n}{r}-1
$$

and

$$
p\left(\alpha-\frac{n}{r}+1\right)>-\frac{n}{r}+1 .
$$


The necessity of the conditions in the above theorems is obtained using ideas from [19]. Our main tools for the sufficient parts will be a off-diagonal Schur's test due to G. O. Okikiolu and integrability conditions of the determinant function among others. We also refer to $[1,20]$ for the corresponding one dimension results and applications.

2.2. Positive Bergman-type operators. In the mixed norm case, following for example the proof of [19, Theorem 1.1] we obtain as consequence of Theorem 2.1, the following result.

Theorem 2.4. Suppose $\nu \in \mathbb{R}, 1<p<\infty$, and $1<q \leq s<\infty$. Assume that $\frac{\nu}{p^{\prime}}+\frac{\mu}{q}>0$. Then the following conditions are equivalent:

(a) The operator $T_{\alpha, \beta, \gamma}^{+}$is bounded from $L_{\nu}^{p, q}(\mathcal{D})$ to $L_{\mu}^{p, s}(\mathcal{D})$.

(b) The parameters satisfy

$$
\gamma=\alpha+\beta+\frac{n}{r}-\frac{\nu}{q}+\frac{\mu}{s}
$$

and

$$
q\left(\beta-\gamma+2 \frac{n}{r}-1\right)-\frac{n}{r}+1<\nu<q(\beta+1)+\frac{n}{r}-1
$$

and

$$
\frac{n}{r}-1-s \alpha<\mu<s(\gamma-\alpha)-\frac{n}{r}+1
$$

In the same way, we have the following first limit case as consequence of Theorem 2.2.

Theorem 2.5. Suppose $\nu \in \mathbb{R}$, and $1<p, s<\infty$. Assume that $\mu>0$. Then the following conditions are equivalent:

(a) The operator $T_{\alpha, \beta, \gamma}^{+}$is bounded from $L_{\nu}^{p, 1}(\mathcal{D})$ to $L_{\mu}^{p, s}(\mathcal{D})$.

(b) The parameters satisfy

$$
\gamma=\alpha+\beta+\frac{n}{r}-\nu+\frac{\mu}{s}
$$

and

$$
\gamma>0
$$

and

$$
\frac{n}{r}-1-s \alpha<\mu<s(\gamma-\alpha)-\frac{n}{r}+1 .
$$

The following second limit case also follows from Theorem 2.3.

Theorem 2.6. Suppose $\nu \in \mathbb{R}$, and $1<p, q<\infty$. Then the following conditions are equivalent:

(a) The operator $T_{\alpha, \beta, \gamma}^{+}$is bounded from $L_{\nu}^{p, q}(\mathcal{D})$ to $L^{p, \infty}(\mathcal{D})$.

(b) The parameters satisfy

$$
\gamma=\alpha+\beta+\frac{n}{r}-\frac{\nu}{q}
$$

and

$$
\nu<q(\beta+1)+\frac{n}{r}-1,
$$


and

$$
q\left(\alpha-\frac{n}{r}+1\right)>-\frac{n}{r}+1
$$

Finally, we have the following which gives sufficient conditions for the boundedness of operators $T_{\alpha, \beta, \gamma}^{+}$from $L_{\nu}^{p}(\mathcal{D})$ to $L_{\mu}^{q}(\mathcal{D})$.

Theorem 2.7. Suppose $\nu \in \mathbb{R}$ and $1<p \leq q<\infty$. Assume that $\frac{\nu+\frac{n}{r}}{p^{\prime}}+\frac{\mu+\frac{n}{r}}{q}>0$, and that the parameters satisfy

$$
\gamma=\alpha+\beta+\frac{n}{r}-\frac{1}{p}\left(\nu+\frac{n}{r}\right)+\frac{1}{q}\left(\mu+\frac{n}{r}\right)
$$

and

$$
\nu<p(\beta+1)+\left(\frac{n}{r}-1\right)\left(1-\frac{p}{q}\right)
$$

and

$$
-q \alpha+\left(\frac{n}{r}-1\right)\left(1+\frac{q}{p^{\prime}}\right)<\mu .
$$

Then the operator $T_{\alpha, \beta, \gamma}^{+}$is bounded from $L_{\nu}^{p}(\mathcal{D})$ to $L_{\mu}^{q}(\mathcal{D})$.

For the proof of Theorem 2.7, we directly appeal to the same off-diagonal Schur's test of G. O. Okikiolu.

Once again, we recall that the boundedness of $T_{\alpha, \beta, \gamma}^{+}$implies the boundedness of $T_{\alpha, \beta, \gamma}$ although the boundedness of $T_{\alpha, \beta, \gamma}$ is expected in a larger range than the one of $T_{\alpha, \beta, \gamma}^{+}$.

Given two positive quantities $A$ and $B$, the notation $A \lesssim B$ (resp. $B \lesssim A$ ) will mean that there is an universal constant $C>0$ such that $A \leq C B$ (resp. $B \leq C A$ ). When $A \lesssim B$ and $B \lesssim A$, we write $A \backsim B$. Notation $C_{\alpha}$ means that the constant $C$ depends on the parameter $\alpha$.

\section{Preliminaries}

We give here some useful tools needed in our presentation. We refer to [13] for the following results. For all vector $\mathbf{s}=\left(s_{1}, \cdots, s_{r}\right)$, we denote

$$
\Delta^{\mathbf{s}}(x)=\Delta_{1}^{s_{1}-s_{2}}(x) \Delta_{2}^{s_{2}-s_{3}}(x) \cdots \Delta_{r}^{s_{r}}(x)
$$

the generalized power of the determinant function. Here $\Delta_{j}(x)$ 's are the principal minors of $x \in \Omega$ with respect to the Jordan algebra structure of the symmetric cone $\Omega$. Also $\Delta_{r}(x):=\Delta(x)$, the determinant of $x$.

Put $(r-1) \frac{d}{2}=\frac{n}{r}-1$. We recall the following result.

Lemma 3.1. Let $v \in \Omega$ and $\mathbf{s}, \mathbf{t} \in \mathbb{C}^{r}$. The integral

$$
\int_{\Omega} \Delta^{\mathbf{s}}(y+v) \Delta^{\mathbf{t}-\frac{n}{r}}(y) d y
$$

converges if for every $j=1, \cdots, r$ we have $\Re t_{j}>(r-j) \frac{d}{2}$ and $\Re\left(s_{j}+t_{j}\right)<-(j-1) \frac{d}{2}$. In this case, we have

$$
\int_{\Omega} \Delta^{\mathbf{s}}(y+v) \Delta^{\mathbf{t}-\frac{n}{r}}(y) d y=C_{\mathbf{s}, \mathbf{t}} \Delta^{\mathbf{s}+\mathbf{t}}(v) .
$$


We also need the following result.

Lemma 3.2. Let $\alpha$ be real. Then the function $f(z)=\Delta^{-\alpha}\left(\frac{z+i t}{i}\right)$, with $t \in \Omega$, belongs to $L_{\nu}^{p, q}(\mathcal{D})$ if and only if $\nu>\frac{n}{r}-1$ and $\alpha>\max \left(\frac{2 \frac{n}{r}-1}{p}, \frac{n}{r p}+\frac{\nu+\frac{n}{r}-1}{q}\right)$. In this case,

$$
\|f\|_{L_{\nu}^{p, q}}^{q}=C_{\alpha, p, q} \Delta^{-q \alpha+\frac{n q}{r p}+\nu}(t) .
$$

Proof. See [4, Lemma 3.20].

The following extension of the Schur's test is due to G. O. Okikiolu [18].

Lemma 3.3. Let $p, r, q$ be positive numbers such that $1<p \leq r$ and $\frac{1}{p}+\frac{1}{q}=1$. Let $K(x, y)$ be a non-negative measurable function on $X \times Y$ and suppose there exist $0<t \leq 1$, measurable functions $\phi_{1}: X \rightarrow(0, \infty), \quad \phi_{2}: Y \rightarrow(0, \infty)$ and nonnegative constants $M_{1}, M_{2}$ such that

$$
\begin{aligned}
\int_{X} K(x, y)^{t q} \phi_{1}^{q}(y) \mathrm{d} \mu(y) & \leq M_{1}^{q} \phi_{2}^{q}(x) \quad \text { a.e on } Y \quad \text { and } \\
\int_{Y} K(x, y)^{(1-t) r} \phi_{2}^{r}(x) \mathrm{d} \nu(x) & \leq M_{2}^{r} \phi_{1}^{r}(y) \quad \text { a.e on } X .
\end{aligned}
$$

If $T$ is given by

$$
T f(x)=\int_{X} f(y) K(x, y) \mathrm{d} \mu(y)
$$

where $f \in L^{p}(X, \mathrm{~d} \mu)$, then $T: L^{p}(X, \mathrm{~d} \mu) \longrightarrow L^{r}(Y, \mathrm{~d} \nu)$ is bounded and for each $f \in$ $L^{p}(X, \mathrm{~d} \mu)$,

$$
\|T f\|_{L^{r}(Y, \mathrm{~d} \nu)} \leq M_{1} M_{2}\|f\|_{L^{p}(X, \mathrm{~d} \mu)} .
$$

The following limit case of Okikiolu result is proved in [21].

Lemma 3.4. Let $\mu$ and $\nu$ be positive measures on the space $X$ and let $K(x, y)$ be nonnegative measurable functions on $X \times Y$. Let $T$ be the integral operator with kernel $K(x, y)$ defined by

$$
T f(x)=\int_{X} f(y) K(x, y) \mathrm{d} \mu(y) .
$$

Suppose $1=p \leq q<\infty$. Let $\gamma$ and $\delta$ be two real numbers such that $\gamma+\delta=1$. If there exist positive functions $h_{1}$ and $h_{2}$ with positive constants $C_{1}$ and $C_{2}$ such that

$$
\begin{gathered}
\text { ess } \sup _{y \in Y} h_{1}(y) K(x, y)^{\gamma} \leq C_{1} h_{2}(x) \quad \text { for almost all } x \in X \quad \text { and } \\
\int_{X} h_{2}(x)^{q} K(x, y)^{\delta q} \mathrm{~d} \nu \leq C_{2} h_{1}(y)^{q} \quad \text { for almost all } y \in Y,
\end{gathered}
$$

then $T$ is bounded from $L^{1}(X, \mathrm{~d} \nu)$ into $L^{q}(X, \mathrm{~d} \nu)$ and the norm of this operator does not exceed $C_{1} C_{2}^{\frac{1}{q}}$. 


\section{Off-Diagonal estimates for the family $S_{\alpha, \beta, \gamma}$}

Let $\Omega$ be an irreducible symmetric cone in $\mathbb{R}^{n}$ of $\operatorname{rank} r$. Let $\alpha, \beta, \gamma, \nu, \mu$ be real parameters. In this section, we obtain necessary and sufficient conditions for the boundedness of the operators $S=S_{\alpha, \beta, \gamma}$ from $L_{\nu}^{p}(\Omega)$ to $L_{\mu}^{q}(\Omega), 1 \leq p \leq q \leq \infty$. We recall that the integral operators $S=S_{\alpha, \beta, \gamma}$ are defined on $\Omega$ by

$$
S g(y)=\Delta^{\alpha}(y) \int_{\Omega} \Delta^{-\gamma}(y+x) g(x) \Delta^{\beta}(x) d x .
$$

This operator has already been considered by the author of [19]. He found necessary and sufficient conditions under which this operator is bounded on $L_{\nu}^{q}(\Omega)$, using the Schur Lemma. He has applied this lemma with vectorial powers of the determinant function. We shall prove here, a more general result, an off-diagonal estimate of this operator. It will require the use of a more general Schur's test result for sufficiency: the Okikiolu's lemma.

4.1. Sufficiency for the boundedness of $S_{\alpha, \beta, \gamma}$. Let us start by proving the following result.

Lemma 4.1. Let $\nu, \mu \in \mathbb{R}$ and $1<p \leq q<\infty$. Assume that $\frac{\nu}{p^{\prime}}+\frac{\mu}{q}>0$. Suppose that the parameters satisfy

$$
\gamma=\alpha+\beta+\frac{n}{r}-\frac{\nu}{p}+\frac{\mu}{q}
$$

and

$$
p\left(\beta-\gamma+2 \frac{n}{r}-1\right)-\frac{n}{r}+1<\nu<p(\beta+1)+\frac{n}{r}-1,
$$

and

$$
\frac{n}{r}-1-q \alpha<\mu<q(\gamma-\alpha)-\frac{n}{r}+1
$$

Then the operator $S$ is bounded from $L_{\nu}^{p}(\Omega)$ into $L_{\mu}^{q}(\Omega)$.

Proof. Assume

$$
\gamma=\alpha+\beta+\frac{n}{r}-\frac{\nu}{p}+\frac{\mu}{q}
$$

and let

$$
\omega=\alpha+\beta-\gamma-\nu+\frac{n}{r}=-\left(\frac{\nu}{p^{\prime}}+\frac{\mu}{q}\right)<0 .
$$

From the left inequality of (28), we have $p\left(\beta-\gamma+2 \frac{n}{r}-1\right)-\frac{n}{r}+1<\nu$ which is equivalent to $\frac{\nu+\frac{n}{r}-1}{p}>\beta-\gamma+2 \frac{n}{r}-1$. Multiplying this last inequality by $\omega<0$ yields $\frac{\nu+\frac{n}{r}-1}{p} \omega<$ $\left(\beta-\gamma+2 \frac{n}{r}-1\right) \omega$ i.e.

$$
\frac{\nu+\frac{n}{r}-1}{p} \omega+\frac{\beta-\gamma+2 \frac{n}{r}-1}{p^{\prime}} \nu<-\frac{\beta-\gamma+2 \frac{n}{r}-1}{q} \mu .
$$

This is equivalent to

$$
\frac{\nu+\frac{n}{r}-1}{p^{\prime}} \omega-\frac{\beta-\gamma-\nu+\frac{n}{r}}{p^{\prime}} \nu>\frac{\beta-\gamma-\nu+\frac{n}{r}}{q} \mu .
$$


From the right inequality of (28), we have $\nu<p(\beta+1)+\frac{n}{r}-1$ which is equivalent to $\frac{\nu-\frac{n}{r}+1}{p}<\beta+1$. Hence, $\nu-\frac{n}{r}+1-\frac{1}{p^{\prime}}\left(\nu-\frac{n}{r}+1\right)<\beta+1$ so that $\beta-\nu+\frac{n}{r}+\frac{1}{p^{\prime}}\left(\nu-\frac{n}{r}+1\right)>0$. Multiplying this last inequality by $\omega$ yields $\left(\beta-\nu+\frac{n}{r}\right) \omega+\frac{1}{p^{\prime}}\left(\nu-\frac{n}{r}+1\right) \omega<0$, i.e.

$$
\frac{\nu-\frac{n}{r}+1}{p^{\prime}} \omega-\frac{\beta-\nu+\frac{n}{r}}{p^{\prime}} \nu<\frac{\beta-\nu+\frac{n}{r}}{q} \mu .
$$

From the left inequality of (29), we have $\frac{n}{r}-1-q \alpha<\mu$ which is equivalent to $-\frac{\mu-\frac{n}{r}+1}{q}-$ $\alpha<0$. Multiplying this last inequality by $\omega$ yields $-\frac{\mu-\frac{n}{r}+1}{q} \omega-\alpha w>0$, i.e.

$$
\frac{\mu-\frac{n}{r}+1}{q} \omega-\alpha \frac{\mu}{q}<\alpha \frac{\nu}{p^{\prime}} .
$$

From the right inequality of (29), we have $\mu<q(\gamma-\alpha)-\frac{n}{r}+1$ which is equivalent to $\frac{\mu+\frac{n}{r}-1}{q}<\gamma-\alpha$. Multiplying this last inequality by $\omega$ yields $\frac{\mu+\frac{n}{r}-1}{q} \omega>(\gamma-\alpha) \omega$, i.e.

$$
\frac{\mu+\frac{n}{r}-1}{q} \omega+\frac{\gamma-\alpha}{q} \mu>-\frac{\gamma-\alpha}{p^{\prime}} \nu .
$$

The inequalities (31), (32), (30) and (33) yield the existence of two real numbers $u$ and $v$ such that

$$
\left\{\begin{array}{l}
\frac{\nu-\frac{n}{r}+1}{p^{\prime}} \omega-\frac{\beta-\nu+\frac{n}{r}}{p^{\prime}} \nu<u \omega+\left(\beta-\nu+\frac{n}{r}\right)(v-u)<\frac{\beta-\nu+\frac{n}{r}}{q} \mu \\
\frac{\mu-\frac{n}{r}+1}{q} \omega-\alpha \frac{\mu}{q}<v \omega+\alpha(u-v)<\alpha \frac{\nu}{p^{\prime}}
\end{array}\right.
$$

and

$$
\left\{\begin{array}{l}
\frac{\nu+\frac{n}{r}-1}{p^{\prime}} \omega-\frac{\beta-\gamma-\nu+\frac{n}{r}}{p^{\prime}} \nu>u \omega+\left(\beta-\gamma-\nu+\frac{n}{r}\right)(v-u)>\frac{\beta-\gamma-\nu+\frac{n}{r}}{q} \mu \\
\frac{\mu+\frac{n}{r}-1}{q} \omega+\frac{\gamma-\alpha}{q} \mu>v \omega+(\gamma-\alpha)(v-u)>-\frac{\gamma-\alpha}{p^{\prime}} \nu .
\end{array}\right.
$$

Now, (34) is equivalent to

$$
\left\{\begin{array}{l}
-\frac{\beta-\nu+\frac{n}{r}}{\omega}\left[-\frac{\mu}{q}-u+v\right]<u<\frac{\nu-\frac{n}{r}+1}{p^{\prime}}+\frac{\beta-\nu+\frac{n}{r}}{\omega}\left[-\frac{\nu}{p^{\prime}}+u-v\right] \\
-\frac{\alpha}{\omega}\left[-\frac{\nu}{p^{\prime}}+u-v\right]<v<\frac{\mu-\frac{n}{r}+1}{q}+\frac{\alpha}{\omega}\left[-\frac{\mu}{q}-u+v\right]
\end{array}\right.
$$

and (35) is equivalent to

$$
\left\{\begin{array}{l}
\frac{\nu+\frac{n}{r}-1}{p^{\prime}}+\frac{\beta-\gamma-\nu+\frac{n}{r}}{\omega}\left[-\frac{\nu}{p^{\prime}}+u-v\right]<u<-\frac{\beta-\gamma-\nu+\frac{n}{r}}{\omega}\left[-\frac{\mu}{q}-u+v\right] \\
\frac{\mu+\frac{n}{r}-1}{q}-\frac{\gamma-\alpha}{\omega}\left[-\frac{\mu}{q}-u+v\right]<v<\frac{\gamma-\alpha}{\omega}\left[-\frac{\nu}{p^{\prime}}+u-v\right] .
\end{array}\right.
$$

Let

$$
t=\frac{-\frac{\nu}{p^{\prime}}+u-v}{\omega}
$$

then

$$
1-t=\frac{-\frac{\mu}{q}-u+v}{w}
$$


Since $\omega<0$, we choose $u$ and $v$ such that $0<v-u<\frac{\mu}{q}$. Thus, we have $0<t<1$. Therefore (36) and (37) become

$$
\left\{\begin{array}{l}
-\left(\beta-\nu+\frac{n}{r}\right)(1-t)<u<\frac{\nu-\frac{n}{r}+1}{p^{\prime}}+\left(\beta-\nu+\frac{n}{r}\right) t \\
-\alpha t<v<\frac{\mu-\frac{n}{r}+1}{q}+\alpha(1-t)
\end{array}\right.
$$

and

$$
\left\{\begin{array}{l}
\frac{\nu+\frac{n}{r}-1}{p^{\prime}}+\left(\beta-\gamma-\nu+\frac{n}{r}\right) t<u<-\left(\beta-\gamma-\nu+\frac{n}{r}\right)(1-t) \\
\frac{\mu+\frac{n}{r}-1}{q}-(\gamma-\alpha)(1-t)<v<(\gamma-\alpha) t
\end{array}\right.
$$

respectively.

We shall now use the Okikiolu test to conclude. To this effect, we observe that the kernel of the operator $S: L_{\nu}^{p}(\Omega) \rightarrow L_{\mu}^{q}(\Omega)$ with respect to the measure $\Delta^{\nu-\frac{n}{r}}(x) d x$ is given by

$$
K(y, x)=\Delta^{\alpha}(y) \Delta^{-\gamma}(y+x) \Delta^{\beta-\nu+\frac{n}{r}}(x) .
$$

Consider the positive functions $\phi_{1}(x)=\Delta^{-u}(x)$ and $\phi_{2}(y)=\Delta^{-v}(y)$. Then

$$
\begin{aligned}
I_{1} & =\int_{\Omega} K(y, x)^{t p^{\prime}} \phi_{1}(x)^{p^{\prime}} \Delta^{\nu-\frac{n}{r}}(x) d x \\
& =\Delta^{t p^{\prime} \alpha}(y) \int_{\Omega} \Delta^{-t p^{\prime} \gamma}(y+x) \Delta^{t p^{\prime}\left(\beta-\nu+\frac{n}{r}\right)-p^{\prime} u+\nu-\frac{n}{r}}(x) d x .
\end{aligned}
$$

The last integral above converges because from the right inequality in (38) involving $u$ and the left inequality in (39) involving $u$, we have $t p^{\prime}\left(\beta-\nu+\frac{n}{r}\right)-p^{\prime} u+\nu>\frac{n}{r}-1$ and $-t p^{\prime} \gamma+t p^{\prime}\left(\beta-\nu+\frac{n}{r}\right)-p^{\prime} u+\nu<-\frac{n}{r}+1$ respectively. It follows using Lemma 3.1 that

$$
\begin{aligned}
I_{1} & =\Delta^{t p^{\prime} \alpha}(y) \int_{\Omega} \Delta^{-t p^{\prime} \gamma}(y+x) \Delta^{t p^{\prime}\left(\beta-\nu+\frac{n}{r}\right)-p^{\prime} u+\nu-\frac{n}{r}}(x) d x \\
& =C_{1} \Delta^{t p^{\prime} \alpha-t p^{\prime} \gamma+t p^{\prime}\left(\beta-\nu+\frac{n}{r}\right)-p^{\prime} u+\nu}(y) \\
& =C_{1} \Delta^{-p^{\prime} v}(y)=C_{1} \phi_{2}(y)^{p^{\prime}}
\end{aligned}
$$

since $t p^{\prime} \alpha-t p^{\prime} \gamma+t p^{\prime}\left(\beta-\nu+\frac{n}{r}\right)-p^{\prime} u+\nu=t p^{\prime} w-p^{\prime} u+\nu=p^{\prime}\left(-\frac{\nu}{p^{\prime}}+u-v\right)-p^{\prime} u+\nu=-p^{\prime} v$.

Now,

$$
\begin{aligned}
I_{2} & =\int_{\Omega} K(y, x)^{(1-t) q} \phi_{2}(y)^{q} d \mu(y) \\
& =\Delta^{(1-t) q\left(\beta-\nu+\frac{n}{r}\right)}(x) \int_{\Omega} \Delta^{-(1-t) q \gamma}(y+x) \Delta^{(1-t) q \alpha-q v+\mu-\frac{n}{r}}(y) d y .
\end{aligned}
$$

The last integral above converges because from the right inequality in (38) involving $v$ and the left inequality in (39) involving $v$, we have

$$
(1-t) q \alpha-q v+\mu>\frac{n}{r}-1 \text { and }-(1-t) q \gamma+(1-t) q \alpha-q v+\mu<-\frac{n}{r}+1
$$


respectively. It follows using Lemma 3.1 again that

$$
\begin{aligned}
I_{2} & =\Delta^{(1-t) q\left(\beta-\nu+\frac{n}{r}\right)}(x) \int_{\Omega} \Delta^{-(1-t) q \gamma}(y+x) \Delta^{(1-t) q \alpha-q v+\mu-\frac{n}{r}}(y) d y \\
& =C_{2} \Delta^{(1-t) q\left(\beta-\nu+\frac{n}{r}\right)-(1-t) q \gamma+(1-t) q \alpha-q v+\mu}(x) \\
& =C_{2} \Delta^{-q u}(x)=C_{2} \phi_{1}(x)^{q}
\end{aligned}
$$

since

$$
\begin{aligned}
& (1-t) q\left(\beta-\nu+\frac{n}{r}\right)-(1-t) q \gamma+(1-t) q \alpha-q v+\mu \\
= & (1-t) q \omega-q v+\mu \\
= & q \omega-q t \omega-q v+\mu=-q \frac{\nu}{p^{\prime}}-\mu-q\left(\frac{-\nu}{p^{\prime}}+u-v\right)-q v+\mu \\
= & -q u .
\end{aligned}
$$

Thus by the Okikiolu test, we conclude that $S: L_{\nu}^{p}(\Omega) \rightarrow L_{\mu}^{q}(\Omega)$ is bounded.

Remark 4.2. One could have also taken the powers $u$ and $v$ in our test functions as vectors, i.e. $\mathbf{u}=\left(u_{1}, \ldots, u_{r}\right)$ and $\mathbf{v}=\left(v_{1}, \ldots, v_{r}\right)$. Indeed, in doing this, Lemma 3.1 again shows that for every $j=1, \ldots, r$, we should have

$$
\left\{\begin{array}{l}
t p^{\prime}\left(\beta-\nu+\frac{n}{r}\right)-p^{\prime} u_{j}+\nu>(r-j) \frac{d}{2} \\
-t p^{\prime} \gamma+t p^{\prime}\left(\beta-\nu+\frac{n}{r}\right)-p^{\prime} u_{j}+\nu<-(j-1) \frac{d}{2}
\end{array}\right.
$$

and

$$
\left\{\begin{array}{l}
(1-t) q \alpha-q v_{j}+\mu>(r-j) \frac{d}{2} \\
-(1-t) q \gamma+(1-t) q \alpha-q v_{j}+\mu<-(j-1) \frac{d}{2}
\end{array} .\right.
$$

But an observation of the systems (38) and (39) shows that $\gamma>0$ so that the interval $\left(\frac{\nu+\frac{n}{r}-1}{p^{\prime}}+\left(\beta-\gamma-\nu+\frac{n}{r}\right) t, \frac{\nu-\frac{n}{r}+1}{p^{\prime}}+\left(\beta-\nu+\frac{n}{r}\right) t\right)$ is not empty; we also see that the interval $\left(\frac{\mu+\frac{n}{r}-1}{q}-(\gamma-\alpha)(1-t), \frac{\mu-\frac{n}{r}+1}{q}+\alpha(1-t)\right)$ is not empty. These facts clearly secure the existence of the vectors $\mathbf{u}$ and $\mathbf{v}$.

The following provides sufficient conditions for the boundedness of $S_{\alpha, \beta, \gamma}$ from $L_{\nu}^{1}(\Omega)$ into $L_{\mu}^{q}(\Omega)$.

Lemma 4.3. Let $\nu, \mu \in \mathbb{R}$ and $1<q<\infty$. Assume that $\mu>0$. Suppose that the parameters satisfy

$$
\gamma=\alpha+\beta+\frac{n}{r}-\nu+\frac{\mu}{q}
$$

and

$$
\gamma>0
$$

and

$$
\frac{n}{r}-1-q \alpha<\mu<q(\gamma-\alpha)-\frac{n}{r}+1
$$

Then the operator $S$ is bounded from $L_{\nu}^{1}(\Omega)$ into $L_{\mu}^{q}(\Omega)$. 
Proof. Assume that $\gamma=\alpha+\beta+\frac{n}{r}-\nu+\frac{\mu}{q}$ and the parameters satisfy (41) and (42). Note that combining (40) and (42), one obtains that

$$
\beta-\gamma+\frac{n}{r}<\nu<\beta+\frac{n}{r}
$$

which corresponds to $(28)$ for $p=1$. Using the notations of the proof of the previous result, we now have

and

$$
\omega=\alpha+\beta+\frac{n}{r}-\nu-\gamma=-\frac{\mu}{q}<0
$$

Now (38) reduces to

$$
t=\frac{u-v}{\omega}
$$

$$
\left\{\begin{array}{l}
-\left(\beta-\nu+\frac{n}{r}\right)(1-t)<u<\left(\beta-\nu+\frac{n}{r}\right) t \\
-\alpha t<v<\frac{\mu-\frac{n}{r}+1}{q}+\alpha(1-t)
\end{array}\right.
$$

and (39) reduces to

$$
\left\{\begin{array}{l}
\left(\beta-\gamma-\nu+\frac{n}{r}\right) t<u<-\left(\beta-\gamma-\nu+\frac{n}{r}\right)(1-t) \\
\frac{\mu+\frac{n}{r}-1}{q}-(\gamma-\alpha)(1-t)<v<(\gamma-\alpha) t
\end{array}\right.
$$

The second condition in Lemma 3.4 can be then checked as in the proof of the previous theorem. Let us also check that the other test condition in the same lemma is verified. That is there are a constant $C>0$ and positive functions $\phi_{1}$ and $\phi_{2}$ such that

$$
\sup _{x \in \Omega} \phi_{1}(x) K(y, x)^{t} \leq C \phi_{2}(y) \text { for almost every } y \in \Omega \text {. }
$$

Our positive functions are still given by $\phi_{1}(x)=\Delta^{-u}(x)$ and $\phi_{2}(y)=\Delta^{-v}(y)$. We then have for every $x \in \Omega$,

$$
\phi_{1}(x) K(y, x)^{t} \phi_{2}(y)^{-1}=\Delta^{-u+t\left(\beta-\nu+\frac{n}{r}\right)}(x) \Delta^{-\gamma t}(y+x) \Delta^{v+t \alpha}(y) .
$$

Observe that from (43), $v+t \alpha>0$. Hence using that for all $a, b \in \Omega$,

$$
\Delta(a+b) \geq \Delta(a)
$$

(see [14]), we obtain that $\Delta^{v+t \alpha}(y) \leq \Delta^{v+t \alpha}(y+x)$ so that

$$
\phi_{1}(x) K(y, x)^{t} \phi_{2}(y)^{-1} \leq \Delta^{-u+t\left(\beta-\nu+\frac{n}{r}\right)}(x) \Delta^{-\gamma t+v+t \alpha}(y+x) .
$$

Moreover, from (43), we have $-u+t\left(\beta-\nu+\frac{n}{r}\right)>0$. Therefore,

$$
\Delta^{-u+t\left(\beta-\nu+\frac{n}{r}\right)}(x) \leq \Delta^{-u+t\left(\beta-\nu+\frac{n}{r}\right)}(y+x) .
$$

It follows that for every $x \in \Omega$,

$$
\phi_{1}(x) K(y, x)^{t} \phi_{2}(y)^{-1} \leq \Delta^{-u+t\left(\beta-\nu+\frac{n}{r}\right)-\gamma t+v+t \alpha}(y+x)=1
$$

for almost every $y \in \Omega$, since

$$
-u+t\left(\beta-\nu+\frac{n}{r}\right)-\gamma t+v+t \alpha=-u+v+t \omega=-u+v+u-v=0 .
$$

We now prove the following. 
Lemma 4.4. Let $\nu \in \mathbb{R}$, and $1<p<\infty$. Suppose that the parameters satisfy

$$
\gamma=\alpha+\beta+\frac{n}{r}-\frac{\nu}{p}
$$

and

$$
\nu<p(\beta+1)+\frac{n}{r}-1
$$

and

$$
p\left(\alpha-\frac{n}{r}+1\right)>-\frac{n}{r}+1 .
$$

Then the operator $S$ is bounded from $L_{\nu}^{p}(\Omega)$ to $L^{\infty}(\Omega)$.

Proof. Let $g \in L_{\nu}^{p}(\Omega)$. Assume that $\gamma=\alpha+\beta+\frac{n}{r}-\frac{\nu}{p}$. Then using the Hölder's inequality and Lemma 3.1, we obtain

$$
\begin{aligned}
|S g(y)| & =\left|\Delta^{\alpha}(y) \int_{\Omega} \Delta^{-\gamma}(y+x) g(x) \Delta^{\beta}(x) d x\right| \\
& =\left|\Delta^{\alpha}(y) \int_{\Omega} \Delta^{-\gamma}(y+x) g(x) \Delta^{\beta-\nu+\frac{n}{r}}(x) d V_{\nu}(x)\right| \\
& \leq \Delta^{\alpha}(y) \int_{\Omega} \Delta^{-\gamma}(y+x)|g(x)| \Delta^{\beta-\nu+\frac{n}{r}}(x) d V_{\nu}(x) \\
& \leq\|g\|_{p, \nu} \Delta^{\alpha}(y)\left(\int_{\Omega} \Delta^{-p^{\prime} \gamma}(y+x) \Delta^{p^{\prime}\left(\beta-\nu+\frac{n}{r}\right)+\nu-\frac{n}{r}}(x) d x\right)^{1 / p^{\prime}} \\
& =C\|g\|_{p, \nu} \Delta^{\alpha-\gamma+\beta-\nu+\frac{n}{r}+\frac{\nu}{p^{\prime}}}(y) \\
& =C\|g\|_{p, \nu} .
\end{aligned}
$$

Hence

$$
\sup _{y \in \Omega}|S g(y)| \leq C\|g\|_{p, \nu}
$$

The proof is complete.

\subsection{Necessity for the boundedness of $S_{\alpha, \beta, \gamma}$.}

Lemma 4.5. Let $\nu, \mu \in \mathbb{R}$ and $1<p \leq q<\infty$. Assume that $\frac{\nu}{p^{\prime}}+\frac{\mu}{q}>0$. If the operator $S$ is bounded from $L_{\nu}^{p}(\Omega)$ into $L_{\mu}^{q}(\Omega)$, then the parameters satisfy the conditions (27), (28) and (29).

Proof. We start with the proof of the homogeneity condition (27), that is,

$$
\gamma=\alpha+\beta+\frac{n}{r}-\frac{\nu}{p}+\frac{\mu}{q}
$$

For this, we recall that the determinant function is homogeneous of degree $r$ (see [14]). Let $R>0$. To any $f \in L^{p}\left(\Omega, \Delta^{\nu-\frac{n}{r}}(y) d y\right)$, we associate the function $f_{R}$ defined by $f_{R}(y)=$ $f(R y)$. One easily checks that

$$
\left\|f_{R}\right\|_{p, \nu}=R^{-r \frac{\nu}{p}}\|f\|_{p, \nu}
$$

An easy change of variable combined with the fact that the determinant function is homogeneous of degree $r$ provides

$$
S\left(f_{R}\right)(y)=R^{-n+r(\gamma-\beta-\alpha)} S f(R y) .
$$


It follows using again the homogeneity of the determinant function that

$$
\left\|S f_{R}\right\|_{q, \mu}=R^{-n+r\left(\gamma-\beta-\alpha-\frac{\mu}{q}\right)}\|S f\|_{q, \mu} .
$$

From the boundedness of the operator $S$, we have that there is a constant $C$ such that for any $f \in L^{p}\left(\Omega, \Delta^{\nu-\frac{n}{r}}(y) d y\right)$,

$$
R^{-n+r\left(\gamma-\beta-\alpha-\frac{\mu}{q}\right)}\|S f\|_{q, \mu}=\left\|S f_{R}\right\|_{q, \mu} \leq C\left\|f_{R}\right\|_{1, \nu}=C R^{-r \frac{\nu}{p}}\|f\|_{p, \nu},
$$

that is,

$$
R^{-n+r\left(\gamma-\beta-\alpha-\frac{\mu}{q}+\frac{\nu}{p}\right)}\|S f\|_{q, \mu} \leq C\|f\|_{p, \nu} .
$$

As the latter holds for every $f \in L^{p}\left(\Omega, \Delta^{\nu-\frac{n}{r}}(y) d y\right)$ and as $R$ was taken arbitrary, we should necessarily have

$$
-n+r\left(\gamma-\beta-\alpha-\frac{\mu}{q}+\frac{\nu}{p}\right)=0
$$

which leads to $\gamma=\alpha+\beta+\frac{n}{r}-\frac{\nu}{p}+\frac{\mu}{q}$. This proves (27).

It is well known that the symmetric cone $\Omega$ induces in $V \equiv \mathbb{R}^{n}$ a structure of Euclidean Jordan algebra. We denote by $\underline{\mathbf{e}}$ the identity element in $V$. Let $g=\chi_{B(\underline{\mathbf{e}}, 1)}$, where $B(\underline{\mathbf{e}}, 1)$ is the Euclidean ball about $\underline{\mathbf{e}}$ with radius 1. Following [19], we have

$$
S g(y) \simeq \Delta^{\alpha}(y) \Delta^{-\gamma}(y+\underline{\mathbf{e}}) .
$$

It follows that if $S$ is bounded from $L^{p}\left(\Omega, \Delta^{\nu-\frac{n}{r}}(y) d y\right)$ to $L^{q}\left(\Omega, \Delta^{\mu-\frac{n}{r}}(y) d y\right)$, then the function $\Delta^{\alpha}(y) \Delta^{-\gamma}(y+\underline{\mathbf{e}})$ is in $L^{q}\left(\Omega, \Delta^{\mu-\frac{n}{r}}(y) d y\right)$, which means that

$$
\int_{\Omega} \Delta^{q \alpha+\mu-\frac{n}{r}}(y) \Delta^{-q \gamma}(y+\mathbf{e}) d y<\infty .
$$

It follows from Lemma 3.1 that we should have

$$
q \alpha+\mu>\frac{n}{r}-1 \quad \text { and } \quad-q \gamma+q \alpha+\mu<-\frac{n}{r}+1 .
$$

That is

$$
\frac{n}{r}-1-q \alpha<\mu<q(\gamma-\alpha)-\frac{n}{r}+1
$$

which is condition (29). To prove the necessity of the condition (28), we proceed by duality. We have that the boundedness of $S$ from $L^{p}\left(\Omega, \Delta^{\nu-\frac{n}{r}}(y) d y\right)$ to $L^{q}\left(\Omega, \Delta^{\mu-\frac{n}{r}}(y) d y\right)$ is equivalent to the boundedness of the adjoint $S^{*}$ of $S$ from $L^{q^{\prime}}\left(\Omega, \Delta^{\mu+q^{\prime}(\nu-\mu)-\frac{n}{r}}(y) d y\right)$ to $L^{p^{\prime}}\left(\Omega, \Delta^{\nu-\frac{n}{r}}(y) d y\right)$. We note that $S^{*}$ is given by

$$
S^{*} g(v)=\Delta^{\beta-\nu+\frac{n}{r}}(v) \int_{\Omega} \Delta^{-\gamma}(y+v) g(y) \Delta^{\alpha+\nu-\frac{n}{r}}(y) d y .
$$

Proceeding as above, we obtain that the function $\Delta^{\beta-\nu+\frac{n}{r}}(v) \Delta^{-\gamma}(v+\underline{\mathbf{e}})$ must belong to $L^{p^{\prime}}\left(\Omega, \Delta^{\nu-\frac{n}{r}}(y) d y\right)$. Using again Lemma 3.1, we see that we must have $\left(\beta-\nu+\frac{n}{r}\right) p^{\prime}+\nu>$ $\frac{n}{r}-1$ and $-p^{\prime} \gamma+\left(\beta-\nu+\frac{n}{r}\right) p^{\prime}+\nu<-\frac{n}{r}+1$, which is equivalent to $\nu<p(\beta+1)+\frac{n}{r}-1$ and $\nu>p\left(\beta-\gamma+2 \frac{n}{r}-1\right)-\frac{n}{r}+1$. This completes the proof of the lemma.

Let us also prove the necessity of the conditions in Lemma 4.3.

Lemma 4.6. Let $\nu, \mu \in \mathbb{R}$ and $1<q<\infty$. Assume that $\mu>0$. If the operator $S$ is bounded from $L_{\nu}^{1}(\Omega)$ into $L_{\mu}^{q}(\Omega)$, then the parameters satisfy the conditions (40), (41) and (42). 
Proof. The necessity of the homogeneity condition

$$
\gamma=\alpha+\beta+\frac{n}{r}-\nu+\frac{\mu}{q}
$$

follows as in the proof of (27). The necessity of the condition (42) follows by taking $p=1$ in the proof of the inequality (29) above. To prove the necessity of the condition (41), we proceed again by duality. We have that the boundedness of $S$ from $L^{1}\left(\Omega, \Delta^{\nu-\frac{n}{r}}(y) d y\right)$ to $L^{q}\left(\Omega, \Delta^{\mu-\frac{n}{r}}(y) d y\right)$ is equivalent to the boundedness of the adjoint $S^{*}$ of $S$ from $L^{q^{\prime}}\left(\Omega, \Delta^{\mu+q^{\prime}(\nu-\mu)-\frac{n}{r}}(y) d y\right)$ to $L^{\infty}(\Omega)$. We have seen that $S^{*}$ is given by

$$
S^{*} g(v)=\Delta^{\beta-\nu+\frac{n}{r}}(v) \int_{\Omega} \Delta^{-\gamma}(y+v) g(y) \Delta^{\alpha+\nu-\frac{n}{r}}(y) d y .
$$

Testing again with the function $g=\chi_{B(\underline{\mathbf{e}}, 1)}$, where $B(\underline{\mathbf{e}}, 1)$ is the Euclidean ball about $\underline{\mathbf{e}}$ with radius 1 , we obtain that the function $\Delta^{\beta-\nu+\frac{n}{r}}(v) \Delta^{-\gamma}(v+\underline{\mathbf{e}})$ should belong to $L^{\infty}(\Omega)$. That is for any $v \in \Omega$,

$$
\Delta^{\beta-\nu+\frac{n}{r}}(v) \Delta^{-\gamma}(v+\underline{\mathbf{e}})<\infty .
$$

We recall that for $a, b \in \Omega, \Delta(a+b) \geq \Delta(a)$. Let us prove that condition (50) implies that $\gamma>0$. Indeed, if this is not the case, that is if $\gamma \leq 0$, then

$$
\Delta^{\beta-\nu+\frac{n}{r}}(v) \Delta^{-\gamma}(v+\underline{\mathbf{e}}) \geq \Delta^{\beta-\gamma-\nu+\frac{n}{r}}(v) .
$$

Hence for every $v \in \Omega$, we must have $\Delta^{\beta-\gamma-\nu+\frac{n}{r}}(v)<\infty$, which is only possible if

$$
\beta-\gamma-\nu+\frac{n}{r}=0
$$

But by (40), $\beta-\gamma-\nu+\frac{n}{r}=0$ only if $\mu=-q \alpha$. This is impossible since the latter used in (42) leads to $\frac{n}{r}-1<0$, which is not true. Hence $\gamma>0$.

We finally prove the following.

Lemma 4.7. Suppose $\nu \in \mathbb{R}$, and $1<p<\infty$. If the operator $S$ is bounded from $L_{\nu}^{p}(\Omega)$ into $L^{\infty}(\Omega)$, then the parameters satisfy the conditions (48), (49) and we have $\gamma=\alpha+\beta+\frac{n}{r}-\frac{\nu}{p}$.

Proof. Again, the necessity of condition $\gamma=\alpha+\beta+\frac{n}{r}-\frac{\nu}{p}$ is obtained as in the previous lemmas. To see that (48) and (49) holds, observe that the boundedness of $S$ from $L^{p}\left(\Omega, \Delta^{\nu-\frac{n}{r}}(y) d y\right)$ to $L^{\infty}(\Omega)$ is equivalent to the boundedness of the adjoint $S^{*}$ of $S$ from $L^{1}\left(\Omega, \Delta^{\nu-\frac{n}{r}}(y) d y\right)$ to $L^{p^{\prime}}\left(\Omega, \Delta^{\nu-\frac{n}{r}}(y) d y\right)$, where $S^{*}$ is given by

$$
S^{*} g(v)=\Delta^{\beta-\nu+\frac{n}{r}}(v) \int_{\Omega} \Delta^{-\gamma}(y+v) g(y) \Delta^{\alpha+\nu-\frac{n}{r}}(y) d y .
$$

The proof is then obtained as in the previous lemmas.

4.3. Proof of Theorem 2.1, Theorem 2.2 and Theorem 2.3. Theorem 2.1 follows from Lemma 4.1 and Lemma 4.5. Theorem 2.2 follows from Lemma 4.3 and Lemma 4.6 while Theorem 2.3 is derived from Lemma 4.4 and Lemma 4.7. 


\section{Off-Diagonal estimates for the family $T_{\alpha, \beta, \gamma}^{+}$}

5.1. The mixed norm case. As said in the presentation of our results, following the proof of [19, Theorem 1.1], one has that Theorem 2.4, Theorem 2.5 and Theorem 2.6 follow respectively from Theorem 2.1, Theorem 2.2 and Theorem 2.3.

As a special case of Theorem 2.4, we have the following which extends the diagonal case result $(q=s)[4$, Theorem 4.3] (see also [19, Corollary 3.6]).

Corollary 5.1. Let $1<p<\infty, 1<q \leq s<\infty$, and assume that $\nu, \mu>\frac{n}{r}-1$. Then the operator $P_{\nu}^{+}$is bounded from $L_{\nu}^{p, q}(\mathcal{D})$ to $L_{\mu}^{p, s}(\mathcal{D})$ if and only if $\frac{\nu}{q}=\frac{\mu}{s}$ and $1+\frac{\frac{n}{r}-1}{\mu}<q<$ $1+\frac{\nu}{\frac{n}{r}-1}$.

If we restrict our problem to the boundedness of the positive Bergman projection $P_{\beta}^{+}$ from $L_{\nu}^{p, 1}(\mathcal{D})$ to $L_{\mu}^{p, s}(\mathcal{D})$ with $s>1$, then we deduce the following from Theorem 2.5.

Corollary 5.2. Let $1<p, s<\infty$, and assume that $\beta, \mu>\frac{n}{r}-1$, and $\nu>0$. Then the following are equivalent.

(a) The operator $P_{\beta}^{+}$is bounded from $L_{\nu}^{p, 1}(\mathcal{D})$ to $L_{\mu}^{p, s}(\mathcal{D})$

(b) The parameters satisfy $s \nu=\mu$ and $\frac{n}{r}-1<s(\beta-\nu)$.

As observed in the second paragraph, the range of boundedness of the positive projection $P_{\beta}^{+}$from $L_{\nu}^{p, q}(\mathcal{D})$ into $L_{\mu}^{p, s}(\mathcal{D})$ is not in general optimal for the boundedness of the projection $P_{\beta}$ (see for example $[7,4,19]$ ). If in the corollary above, we choose $\beta$ large enough, we obtain that the optimal condition for the boundedness of $P_{\beta}^{+}$is also optimal for $P_{\beta}$. In particular, putting $\beta=\nu+\frac{n}{r}-1$, we have the following full characterization for the Bergman projection $P_{\beta}$.

Proposition 5.3. Let $1<p<\infty, 1<s<\infty$, and assume that $\mu>\frac{n}{r}-1$ and $\nu>0$. Then the following are equivalent.

(a) The operator $P_{\nu+\frac{n}{r}-1}$ is bounded from $L_{\nu}^{p, 1}(\mathcal{D})$ to $L_{\mu}^{p, s}(\mathcal{D})$

(b) The operator $P_{\nu+\frac{n}{r}-1}^{+}$is bounded from $L_{\nu}^{p, 1}(\mathcal{D})$ to $L_{\mu}^{p, s}(\mathcal{D})$

(c) The parameters satisfy $s \nu=\mu$.

Proof. We already know that the implication (b) $\Rightarrow(\mathrm{a})$ holds. That $(\mathrm{c}) \Rightarrow(\mathrm{b})$ is a special case of Corollary 5.2. That $(\mathrm{a}) \Rightarrow(\mathrm{c})$ can be proved as in Lemma 4.6.

In the diagonal case, it is known that the Bergman projection $P_{\gamma}$ cannot be bounded on $L_{\nu}^{p, q}(\mathcal{D})$ for $q$ larger than $\tilde{q}_{\nu, p}$, where $\tilde{q}_{\nu, p}=\frac{\nu+\frac{n}{r}-1}{\left(\frac{n}{r p^{\prime}}-1\right)_{+}}$with $\tilde{q}_{\nu, p}=\infty$ if $n / r \leq p^{\prime}$ (see $[7,5,4,19])$. In the off-diagonal case, the same phenomenon is observed. Indeed we have the following.

Proposition 5.4. Let $\mu, \nu, \gamma \in \mathbb{R}$, and $1 \leq p<\infty$ and $1 \leq q \leq s<\infty$. If $P_{\gamma}$ extends as a bounded operator from $L_{\nu}^{p, q}(\mathcal{D})$ into $L_{\mu}^{p, s}(\mathcal{D})$, then $B_{\mu}(z, i \underline{\mathbf{e}}) \in L_{\mu}^{p, s}$ and $\Delta^{\gamma-\nu}(\Im z) B_{\mu}(z, i \underline{\mathbf{e}}) \in$ $L_{\nu}^{p^{\prime}, q^{\prime}}$. The latter is equivalent to the following conditions: $\mu>\frac{n}{r}-1,\left(\gamma+\frac{n}{r}\right)>\left(2 \frac{n}{r}+1\right) \max \left\{\frac{1}{p}, \frac{1}{p^{\prime}}\right\}$, and $s>\frac{\mu+\frac{n}{r}-1}{\left(\gamma+\frac{n}{r p^{\prime}}\right)_{+}}$and

$$
\frac{\nu-\frac{n}{r}+1}{\left(\gamma-\frac{n}{r}+1\right)_{+}}<q<\tilde{q}_{\nu, p} .
$$


Proof. Let us denote by $P_{\gamma}^{*}$ the adjoint operator of $P_{\gamma}$ with respect to the pairing $\langle,\rangle_{\nu}$. One easily checks that

$$
P_{\gamma}^{*} f(z)=\Delta^{\gamma-\nu}(\Im z) \int_{\mathcal{D}} B_{\gamma}(z, w) f(w) \Delta^{\nu-n / r}(\Im w) d V(w)
$$

$f \in L_{\mu+(\nu-\mu) s^{\prime}-\frac{n}{r}}^{p^{\prime}, s^{\prime}}(\mathcal{D})$. Let $B_{1}(\underline{i} \underline{\mathbf{e}})$ be the Euclidean ball of radius 1 centered at $i \underline{\mathbf{e}}$. Testing $P_{\gamma}$ with $f_{1}(z)=\chi_{B_{1}(i \mathbf{e})}(z) \Delta^{-\gamma+\frac{n}{r}}(\Im z)$ and $P_{\mu}^{*}$ with $f_{2}(z)=\chi_{B_{1}(i \mathbf{e})}(z) \Delta^{-\nu+\frac{n}{r}}(\Im z)$, we obtain with the help of the mean value property that $P_{\gamma} f_{1}(z)=C B_{\gamma}(z, i \underline{\mathbf{e}})$ and $P_{\gamma}^{*} f_{2}(z)=$ $C \Delta^{\gamma-\nu}(\Im z) B_{\gamma}(z, i \underline{\mathbf{e}})$. It follows that we should have $B_{\gamma}(z, i \underline{\mathbf{e}}) \in L_{\mu}^{p, s}$ and $\Delta^{\gamma-\nu}(\Im z) B_{\gamma}(z, i \underline{\mathbf{e}}) \in$ $L_{\nu}^{p^{\prime}, q^{\prime}}$. By Lemma 3.2 this is equivalent to

and

$$
\begin{gathered}
\mu>\frac{n}{r}-1, \nu+(\gamma-\nu) q^{\prime}>\frac{n}{r}-1, \\
\gamma+\frac{n}{r}>\left(2 \frac{n}{r}-1\right) \max \left(\frac{1}{p^{\prime}}, \frac{1}{p}\right)
\end{gathered}
$$

$$
\gamma+\frac{n}{r}>\max \left\{\frac{n}{r p^{\prime}}+\frac{\nu+(\gamma-\nu) q^{\prime}+\frac{n}{r}-1}{q^{\prime}}, \frac{n}{r p}+\frac{\mu+\frac{n}{r}-1}{s}\right\} .
$$

That is, $\mu>\frac{n}{r}-1, \gamma+\frac{n}{r}>\left(2 \frac{n}{r}-1\right) \max \left(\frac{1}{p^{\prime}}, \frac{1}{p}\right)$, and $s>\frac{\mu+\frac{n}{r}-1}{\left(\gamma+\frac{n}{r p^{\prime}}\right)_{+}}$, and $\frac{\nu-\frac{n}{r}+1}{\left(\gamma-\frac{n}{r}+1\right)_{+}}<q<$ $\tilde{q}_{\nu, p}$

5.2. Proof of Theorem 2.7. We appeal again to Okikiolu result and follow the ideas in the proof of Theorem 2.1.

Proof of Theorem 2.7. As said, we follow the proof of Theorem 2.1. Recall that

$$
\gamma=\alpha+\beta+\frac{n}{r}-\frac{1}{p}\left(\nu+\frac{n}{r}\right)+\frac{1}{q}\left(\mu+\frac{n}{r}\right)
$$

We put

$$
\omega=\alpha+\beta-\gamma-\nu=-\left[\frac{\nu+\frac{n}{r}}{p^{\prime}}+\frac{\mu+\frac{n}{r}}{q}\right]<0 .
$$

The inequality (23) is equivalent to $\frac{\nu-\frac{n}{r}+1}{p}+\frac{1}{q}\left(\frac{n}{r}-1\right)<\beta+1$. Hence,

$$
\beta-\nu+\frac{n}{r}+\frac{1}{p^{\prime}}\left(\nu-\frac{n}{r}+1\right)-\frac{1}{q}\left(\frac{n}{r}-1\right)>0 .
$$

Multiplying this last inequality by $\omega<0$ yields

$$
\left(\beta-\nu+\frac{n}{r}\right) \omega+\frac{1}{p^{\prime}}\left(\nu-\frac{n}{r}+1\right) \omega-\frac{\omega}{q}\left(\frac{n}{r}-1\right)<0,
$$

i.e.

$$
\frac{\nu-\frac{n}{r}+1}{p^{\prime}} \omega-\frac{\beta-\nu+\frac{n}{r}}{p^{\prime}}\left(\nu+\frac{n}{r}\right)<\frac{\beta-\nu+\frac{n}{r}}{q}\left(\mu+\frac{n}{r}\right)+\frac{\omega}{q}\left(\frac{n}{r}-1\right) .
$$

From the inequality (24), we have $\frac{\mu-\frac{n}{r}+1}{q}>-\alpha+\frac{1}{p^{\prime}}\left(\frac{n}{r}-1\right)$. Multiplying this last inequality by $\omega$ yields $\frac{\mu-\frac{n}{r}+1}{q} \omega<-\alpha \omega+\frac{\omega}{p^{\prime}}\left(\frac{n}{r}-1\right)$, i.e.

$$
\frac{\mu-\frac{n}{r}+1}{q} \omega-\alpha \frac{\mu+\frac{n}{r}}{q}<\alpha \frac{\nu+\frac{n}{r}}{p^{\prime}}+\frac{\omega}{p^{\prime}}\left(\frac{n}{r}-1\right) .
$$


The inequalities (51) and (52) yield the existence of two real numbers $u$ and $v$ such that

$$
\left\{\begin{aligned}
& \frac{\nu-\frac{n}{r}+1}{p^{\prime}} \omega-\frac{\beta-\nu+\frac{n}{r}}{p^{\prime}}\left(\nu+\frac{n}{r}\right)<u \omega+\left(\beta-\nu+\frac{n}{r}\right)(v-u) \\
&<\frac{\beta-\nu+\frac{n}{r}}{q}\left(\mu+\frac{n}{r}\right)+\frac{\omega}{q}\left(\frac{n}{r}-1\right) \\
& \frac{\mu-\frac{n}{r}+1}{q} \omega-\alpha \frac{\mu+\frac{n}{r}}{q}<v \omega+\alpha(u-v)< \alpha \frac{\nu+\frac{n}{r}}{p^{\prime}}+\frac{\omega}{p^{\prime}}\left(\frac{n}{r}-1\right)
\end{aligned}\right.
$$

Now, (53) is equivalent to

$$
\left\{\begin{array}{l}
-\frac{\beta-\nu+\frac{n}{r}}{\omega}\left[-\frac{\mu+\frac{n}{r}}{q}-u+v\right]+\frac{1}{q}\left(\frac{n}{r}-1\right)<u<\frac{\nu-\frac{n}{r}+1}{p^{\prime}}+\frac{\beta-\nu+\frac{n}{r}}{\omega}\left[-\frac{\nu+\frac{n}{r}}{p^{\prime}}+u-v\right] \\
-\frac{\alpha}{\omega}\left[-\frac{\nu+\frac{n}{r}}{p^{\prime}}+u-v\right]+\frac{1}{p^{\prime}}\left(\frac{n}{r}-1\right)<v<\frac{\mu-\frac{n}{r}+1}{q}+\frac{\alpha}{\omega}\left[-\frac{\mu+\frac{n}{r}}{q}-u+v\right] .
\end{array}\right.
$$

Let

$$
t=\frac{-\frac{\nu+\frac{n}{r}}{p^{\prime}}+u-v}{\omega}
$$

then

$$
1-t=\frac{-\frac{\mu+\frac{n}{r}}{q}-u+v}{w} .
$$

Since $\omega<0$, we choose $u$ and $v$ such that $0<v-u<\frac{\mu+\frac{n}{r}}{q}$. Thus, we have $0<t<1$. Moreover, (54) becomes

$$
\left\{\begin{array}{l}
-\left(\beta-\nu+\frac{n}{r}\right)(1-t)+\frac{1}{q}\left(\frac{n}{r}-1\right)<u<\frac{\nu-\frac{n}{r}+1}{p^{\prime}}+\left(\beta-\nu+\frac{n}{r}\right) t \\
-\alpha t+\frac{1}{p^{\prime}}\left(\frac{n}{r}-1\right)<v<\frac{\mu-\frac{n}{r}+1}{q}+\alpha(1-t) .
\end{array}\right.
$$

We now observe that the positive kernel of the operator $T^{+}$with respect to $\Delta^{\nu-\frac{n}{r}}(\Im w) d V(w)$ is given by

$$
K(z, w)=\Delta^{\alpha}(\Im z)\left|\Delta^{-\gamma-\frac{n}{r}}\left(\frac{z-\bar{w}}{i}\right)\right| \Delta^{\beta-\nu+\frac{n}{r}}(\Im w) .
$$

Let us consider the following positive functions $\psi_{1}(w)=\Delta^{-u}(\Im w)$ and $\psi_{2}(z)=\Delta^{-v}(\Im z)$. Then

$$
\begin{aligned}
J_{1} & =\int_{\mathcal{D}} K(z, w)^{t p^{\prime}} \psi_{1}(w)^{p^{\prime}} d V_{\nu}(w) \\
& =\Delta^{t p^{\prime} \alpha}(\Im z) \int_{\mathcal{D}}\left|\Delta^{-t p^{\prime}\left(\gamma+\frac{n}{r}\right)}\left(\frac{z-\bar{w}}{i}\right)\right| \Delta^{t p^{\prime}\left(\beta-\nu+\frac{n}{r}\right)-p^{\prime} u+\nu-\frac{n}{r}}(\Im w) d V(w) .
\end{aligned}
$$


The last integral above converges because from the right inequality in (55) involving $u$, we have $t p^{\prime}\left(\beta-\nu+\frac{n}{r}\right)-p^{\prime} u+\nu>\frac{n}{r}-1$ and also

$$
\begin{aligned}
I & :=-t p^{\prime}\left(\gamma+\frac{n}{r}\right)+t p^{\prime}\left(\beta-\nu+\frac{n}{r}\right)-p^{\prime} u+\nu \\
& =-t p^{\prime}(\gamma-\beta+\nu)-p^{\prime} u+\nu \\
& =-t p^{\prime}\left(\alpha+\nu+\frac{n}{r}-\frac{1}{p}\left(\nu+\frac{n}{r}\right)+\frac{1}{q}\left(\mu+\frac{n}{r}\right)\right)-p^{\prime} u+\nu \\
& =-t p^{\prime}(\alpha-\omega)-p^{\prime} u+\nu \\
& =-\alpha t p^{\prime}+t \omega p^{\prime}-p^{\prime} u+\nu \\
& =-\alpha t p^{\prime}+p^{\prime}\left(-\frac{1}{p^{\prime}}\left(\nu+\frac{n}{r}\right)+u-v\right)-p^{\prime} u+\nu \\
& =-\alpha t p^{\prime}-p^{\prime} v-\frac{n}{r}<-2 \frac{n}{r}+1
\end{aligned}
$$

where the last inequality above follows from the left inequality in (55) involving $v$. It follows using Lemma 3.2 that

$$
\begin{aligned}
J_{1} & =\Delta^{t p^{\prime} \alpha}(\Im z) \int_{\mathcal{D}}\left|\Delta^{-t p^{\prime}\left(\gamma+\frac{n}{r}\right)}\left(\frac{z-\bar{w}}{i}\right)\right| \Delta^{t p^{\prime}\left(\beta-\nu+\frac{n}{r}\right)-p^{\prime} u+\nu-\frac{n}{r}}(\Im w) d V(w) \\
& =C_{1} \Delta^{t p^{\prime} \alpha-t p^{\prime}\left(\gamma+\frac{n}{r}\right)+t p^{\prime}\left(\beta-\nu+\frac{n}{r}\right)-p^{\prime} u+\nu+\frac{n}{r}}(\Im z) \\
& =C_{1} \Delta^{-p^{\prime} v}(\Im z)=C_{1} \phi_{2}(z)^{p^{\prime}}
\end{aligned}
$$

Next,

$$
\begin{aligned}
J_{2} & =\int_{\mathcal{D}} K(z, w)^{(1-t) q} \psi_{2}(z)^{q} d V_{\mu}(z) \\
& =\Delta^{(1-t) q\left(\beta-\nu+\frac{n}{r}\right)}(\Im w) \int_{\mathcal{D}}\left|\Delta^{-(1-t) q\left(\gamma+\frac{n}{r}\right)}\left(\frac{z-\bar{w}}{i}\right)\right| \Delta^{(1-t) q \alpha-q v+\mu-\frac{n}{r}}(\Im z) d V(z) .
\end{aligned}
$$

The last integral above converges since from the right inequality in (55) involving $v$, we have $(1-t) q \alpha-q v+\mu>\frac{n}{r}-1$ and also, we have

$$
\begin{aligned}
I & :=-(1-t) q\left(\gamma+\frac{n}{r}\right)+(1-t) q \alpha-q v+\mu \\
& =-(1-t) q\left(\gamma-\alpha+\frac{n}{r}\right)-q v+\mu \\
& =-(1-t) q\left[\beta+2 \frac{n}{r}-\frac{1}{p}\left(\nu+\frac{n}{r}\right)+\frac{1}{q}\left(\mu+\frac{n}{r}\right)\right]-q v+\mu \\
& =-(1-t) q\left[\beta-\nu+\frac{n}{r}+\frac{1}{p^{\prime}}\left(\nu+\frac{n}{r}\right)+\frac{1}{q}\left(\mu+\frac{n}{r}\right)\right]-q v+\mu \\
& =-(1-t) q\left[\beta-\nu+\frac{n}{r}-\omega\right]-q v+\mu \\
& =(1-t) q \omega-(1-t) q\left(\beta-\nu+\frac{n}{r}\right)-q v+\mu \\
& =q\left(-\frac{1}{q}\left(\mu+\frac{n}{r}\right)-u+v\right)-(1-t) q\left(\beta-\nu+\frac{n}{r}\right)-q v+\mu \\
& =-q u-(1-t) q\left(\beta-\nu+\frac{n}{r}\right)-\frac{n}{r}<-2 \frac{n}{r}+1 .
\end{aligned}
$$


where the last inequality above follows from the left inequality in (55) involving $u$. It follows from Lemma 3.2 that

$$
\begin{aligned}
J_{2} & =\Delta^{(1-t) q\left(\beta-\nu+\frac{n}{r}\right)}(\Im w) \int_{\mathcal{D}}\left|\Delta^{-(1-t) q\left(\gamma+\frac{n}{r}\right)}\left(\frac{z-\bar{w}}{i}\right)\right| \Delta^{(1-t) q \alpha-q v+\mu-\frac{n}{r}}(\Im z) d V(z) \\
& =C_{2} \Delta^{(1-t) q\left(\beta-\nu+\frac{n}{r}\right)-(1-t) q\left(\gamma+\frac{n}{r}\right)+(1-t) q \alpha-q v+\mu+\frac{n}{r}}(\Im w) \\
& =C_{2} \Delta^{-q u}(\Im w)=C_{2} \phi_{1}(w)^{q}
\end{aligned}
$$

Thus by the Okikiolu test, we conclude that $T^{+}: L_{\nu}^{p}(\mathcal{D}) \rightarrow L_{\mu}^{q}(\mathcal{D})$ is bounded.

\section{REFERENCES}

[1] J. S. Bansah And B. F. Sehba, Boundedness of a family of Hilbert-type operators and its Bergman-type analogue. Illinois J. Math. 59, no. 4 (2015), 949-977.

[2] BÉkollé, D., A. Bonami, Estimates for the Bergman and Szegő projections in two symmetric domains of $\mathbb{C}^{n}$. Colloq. Math. 68 (1995), 81-100.

[3] BÉkollé, D., A. Bonami, Analysis on tube domains over light cones: some extensions of recent results. Actes des Rencontres d'Analyse Complexe, Poitiers 1999. Éd.. Atlantique et ESA CNRS 6086 (2000), pp. 17-37.

[4] D. Békollé, A. Bonami, G. Garrigós, C. Nana, M. Peloso and F. Ricci, Lecture notes on Bergman projectors in tube domains over cones: an analytic and geometric viewpoint. IMHOTEP 5 (2004), Exposé I, Proceedings of the International Workshop in Classical Analysis, Yaoundé 2001.

[5] D. Békollé, A. Bonami, G. Garrigós and F. Ricci. Littlewood-Paley decompositions related to symmetric cones and Bergman projections in tube domains. Proc. London Math. Soc. 89 (2004), 317360.

[6] D. Békollé, A. Bonami, G. Garrigós, F.Ricci and B. Sehba, Hardy-type inequalities and analytic Besov spaces in tube domains over symmetric cones J. Reine Angew. Math. 647 (2010), 25-56.

[7] D. Békollé, A. Bonami, M. Peloso and F. Ricci, Boundedness of weighted Bergman projections on tube domains over light cones. Math. Z. 237 (2001), 31-59

[8] D. Békollé, J. Gonessa, And C. NAnA, Lebesgue Mixed Norm Estimates for Bergman Projectors: From Tube Domains over Homogeneous Cones to Homogeneous Siegel Domains of Type II, available at https://arxiv.org/abs/1703.07854.

[9] Békollé D. and C. Nana, " $L^{p}$-boundedness of Bergman projections in the tube domain over Vinberg's cone", Journal of Lie Theory Vol. 17 (2007), No 1, 115-144.

[10] A. Bonami, Three related problems on Bergman spaces over symmetric cones. Rend. Mat. Acc. Lincei s. 9, v. 13 (2002), 183-197.

[11] A. Bonami And C. Nana, Some questions related to the Bergman projection in Symmetric domains. Adv. Pure Appl. Math. 6, no. 4, 191-197 (2015).

[12] Bourgain And Demeter, The proof of the $l^{2}$-decoupling conjecture. Ann. of Math. 182, no. 1 (2015), $351-389$

[13] D. Debertol, Besov spaces and boundedness of weighted Bergman projections over symmetric tube domains. Dottorato di Ricerca in Matematica, Università di Genova, Politecnico di Torino, (April 2003).

[14] J. Faraut, A. Korányi, Analysis on symmetric cones. Clarendon Press, Oxford, 1994.

[15] G. Garrigós, A. Seeger, Plate decompositions for cone multipliers. Proc. "Harmonic Analysis and its Applications at Sapporo 2005", Miyachi \& Tachizawa Ed. Hokkaido University Report Series 103, pp. $13-28$.

[16] C. NAna, $L^{p, q}$-Boundedness of Bergman Projections in Homogeneous Siegel Domains of Type II, J. Fourier Anal. Appl. 19 (2013), 997-1019.

[17] C. Nana C. And B. Trojan, " $L^{p}$-Boundedness of Bergman projections in tube domains over homogeneous cones," Ann. Scuola Norm. Sup. Pisa Cl. Sci. (5) Vol. X (2011), 477-511.

[18] G. O. Okikiolu, On Inequalities for Integral Operators. Glasgow Math. J. 11 no. 2 (1969), $126-133$.

[19] B. F. SEhbA "Bergman type operators in tubular domains over symmetric cones", Proc. Edin. Math. Soc. 52 (2) (2009), 529-544.

[20] B. F. SEHBA, Sharp off-diagonal weighted norm estimates for the Bergman projection, available at https://arxiv.org/abs/1703.00275. 
[21] R. ZhaO, Generalization of Schur's Test and its Application to a Class of Integral Operators on the Unit Ball of $C^{n}$. Integr. Equ. Oper. Theo. 82, Issue 4 (2015), 519-532.

Department of Mathematics, Faculty of Science, University of Buea, P. O. Box 63,, Buea CAMEROON

E-mail address: nana.cyrille@ubuea.cm

Department of Mathematics, University of Ghana,, P. O. Box LG 62 Legon, Accra, Ghana

E-mail address: bfsehba@ug.edu.gh 